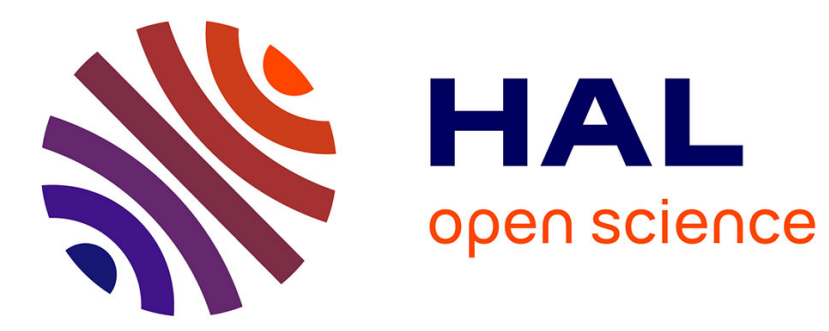

\title{
Multi sub-band Monte Carlo simulation of an ultra-thin double gate MOSFET with 2D electron gas
}

\author{
Jérôme Saint-Martin, A. Bournel, F Monsef, C. Chassat, P. Dollfus
}

\section{To cite this version:}

Jérôme Saint-Martin, A. Bournel, F Monsef, C. Chassat, P. Dollfus. Multi sub-band Monte Carlo simulation of an ultra-thin double gate MOSFET with 2D electron gas. Semiconductor Science and Technology, 2006, 21 (4), pp.L29 - L31. 10.1088/0268-1242/21/4/L01 . hal-01909484

\section{HAL Id: hal-01909484 \\ https://hal.science/hal-01909484}

Submitted on 17 Jul 2019

HAL is a multi-disciplinary open access archive for the deposit and dissemination of scientific research documents, whether they are published or not. The documents may come from teaching and research institutions in France or abroad, or from public or private research centers.
L'archive ouverte pluridisciplinaire HAL, est destinée au dépôt et à la diffusion de documents scientifiques de niveau recherche, publiés ou non, émanant des établissements d'enseignement et de recherche français ou étrangers, des laboratoires publics ou privés. 
RAPID COMMUNICATION

Multi sub-band Monte Carlo simulation of an ultrathin double gate MOSFET with 2D electron gas

To cite this article: J Saint-Martin et al 2006 Semicond. Sci. Technol. 21 L29

View the article online for updates and enhancements.
Related content

An improved empirical approach to introduce quantization effects in the transport direction in multi-subband Monte Carlo simulations

P Palestri, L Lucci, S Dei Tos et al.

- Ultra-short n-MOSFET with strained Si: device performance and ballistic transport Valérie Aubry-Fortuna, Arnaud Bournel, Philippe Dollfus et al.

- Impact of technology scaling on analog and RF performance of SOI-TFET

P Kumari, S Dash and G P Mishra

\section{Recent citations}

Multi-Subband Ensemble Monte Carlo simulations of scaled GAA MOSFETs

L. Donetti et al

- L. Donetti et al

- L. Donetti et al

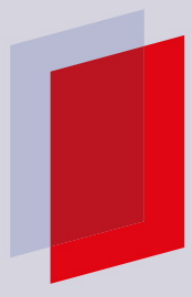

\section{IOP ebooks}

Bringing you innovative digital publishing with leading voices to create your essential collection of books in STEM research. Start exploring the collection - download the first chapter of every title for free. 


\title{
RAPID COMMUNICATION
}

\section{Multi sub-band Monte Carlo simulation of an ultra-thin double gate MOSFET with 2D electron gas}

\author{
J Saint-Martin, A Bournel, F Monsef, C Chassat and P Dollfus \\ Institut d'Electronique Fondamentale, CNRS UMR 8622, Université Paris Sud, Bât. 220, \\ F-91405 Orsay cedex, France \\ E-mail: jerome.saint-martin@ief.u-psud.fr
}

Received 17 November 2005, in final form 18 November 2005

Published 21 February 2006

Online at stacks.iop.org/SST/21/L29

\begin{abstract}
A new two-dimensional self-consistent Monte Carlo simulator including multi sub-band transport in 2D electron gas is described and applied to thin-film SOI double gate MOSFETs. This approach takes into account both out of equilibrium transport and quantization effects. Our method allows us to significantly improve microscopic insight into the operation of deep sub-100 nm CMOS devices. We compare and analyse the results obtained with and without quantization effects for a $15 \mathrm{~nm}$ long DGMOS transistor.
\end{abstract}

(Some figures in this article are in colour only in the electronic version)

\section{Introduction}

Double gate (DG) structures are promising architectures likely to overcome short channel effects in a nanometre scaled MOSFET. Using a form factor $L_{\mathrm{G}} / T_{\mathrm{Si}}$ greater than 3 , where $L_{\mathrm{G}}$ is the gate length and $T_{\mathrm{Si}}$ is the $\mathrm{Si}$ body thickness between the two gate stacks, good performances in both off- and onstates may be obtained without the need of sub-nanometre equivalent oxide thickness [1]. Thus, in sub-50 nm MOSFETs, $T_{\mathrm{Si}}$ should be typically less than $10 \mathrm{~nm}$ and quantization effects in the transverse direction are expected to be more and more significant. Quantization effects may degrade charge control [2] and modify carrier transport [3, 4]. Besides, in nanotransistors where the channel length is comparable to the electron mean free path, quasi-ballistic effects are also known to strongly influence the device performance [5]. However, scattering phenomena may not be neglected in any part of the channel [5]. Therefore, to design a MOSFET suitable for the next CMOS generations, a transport model in which both scatterings and quantum vertical confinement in the channel are accurately taken into account is highly desirable.

In this context, we present results obtained using Monte Carlo simulation of a DG MOSFET self-consistently coupled with the Schrödinger equation in order to properly include quantization effects. For the first time, results obtained from this $2 \mathrm{Dk}$ simulator are compared to those resulting from a classical Monte Carlo approach (3Dk).

\section{Model}

\subsection{The simulator principle}

The simulator principle is inspired by both the mode-space approach of quantum transport [6] and the MC technique developed in [7]. In our model, the $x$-axis, along which the carrier movement is supposed to be semi-classical, is separated from the $z$-axis, along which the energy is quantized in the whole device. For each $x_{0}$-slice of the channel schematized in figure 1, the time-independent 1D Schrödinger equation along the $z$-axis is solved, which provides the eigenenergies $E_{n}$ and wavefunctions $\xi_{n}(z)$ associated with all sub-bands numbered ' $n$ '.

\subsection{Self-consistent Monte Carlo simulation}

During each time step, the classical transport equation of the 2Dk electron gas is solved along the $x$-axis by a Monte Carlo algorithm as summarized in figure 2. According to the mode-space approach, the electric field undergone by a 


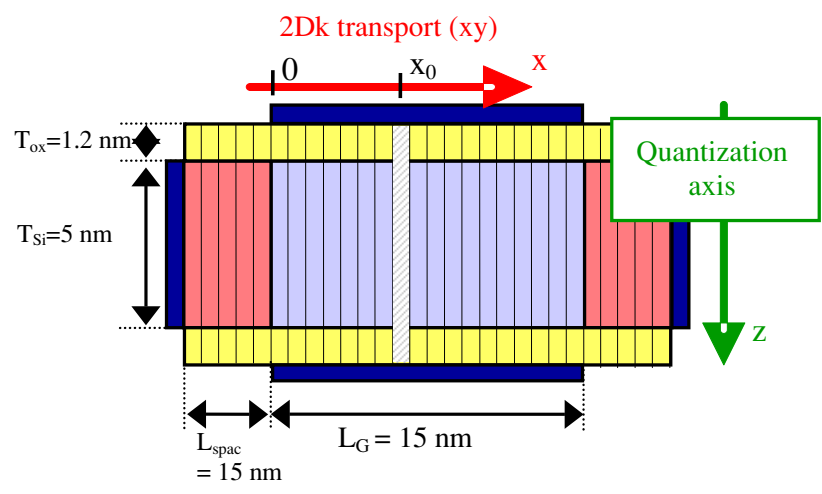

Figure 1. Schematic of the DGMOS structure.

carrier belonging to sub-band ' $n$ ' is given by the $x$-derivative of the associated energy $E_{n}(x)$. This transport algorithm provides the number of electrons present in each sub-band of the 'Schrödinger' $x_{0}$-slice at the end of the time step. The electron density is then calculated by distributing the charge of each electron along its $x_{0}$-slice according to the appropriate probability density $\left|\xi_{n}\left(x_{0}, z\right)\right|^{2}$. Next, a new loop can start from a new 2D Poisson's equation solution followed by a 1D Schrödinger's equation solution, and so on. By this method, the 1D Schrödinger, 2D Poisson and Boltzmann equations are solved self-consistently.

\section{3. $2 D$ scattering mechanisms}

Scattering mechanisms included in the simulation are bulk phonon (acoustic intra-valley and intervalley phonons as described in [8]) and impurity scatterings, taking nonparabolic and ellipsoidal band structures into account. Details on the calculation of $2 \mathrm{Dk}$ scattering rates may be found in [9]. The same phonon coupling constants are used for the $2 \mathrm{Dk}$ and $3 \mathrm{Dk}$ electron gases. In order to facilitate the comparison between $2 \mathrm{Dk}$ and $3 \mathrm{Dk}$ simulations, roughness scattering is not included in the present work.

\section{Results}

The simulated DGMOS device, described in figure 1, has a gate length $L_{\mathrm{G}}$ equal to $15 \mathrm{~nm}$. The $\mathrm{SiO}_{2}$ gate oxide $T_{\text {ox }}$

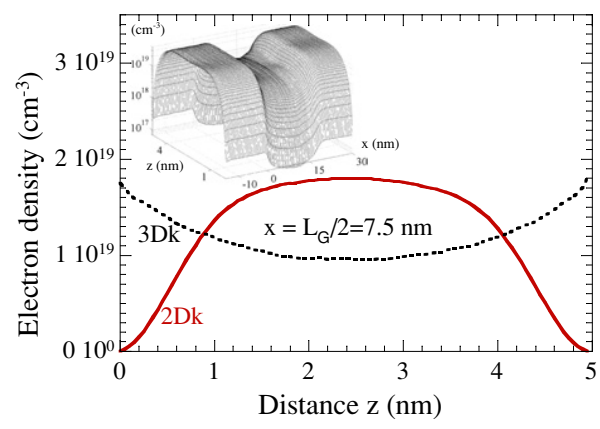

Figure 3. Electron density versus distance $z$ perpendicular to the gate stack in the middle of the channel $\left(x=L_{\mathrm{G}} / 2\right)$ at the on-state $\left(V_{\mathrm{GS}}=V_{\mathrm{DS}}=V_{\mathrm{DD}}\right)$. Inset: $2 \mathrm{D}$ cartography of electron density at the on-state.

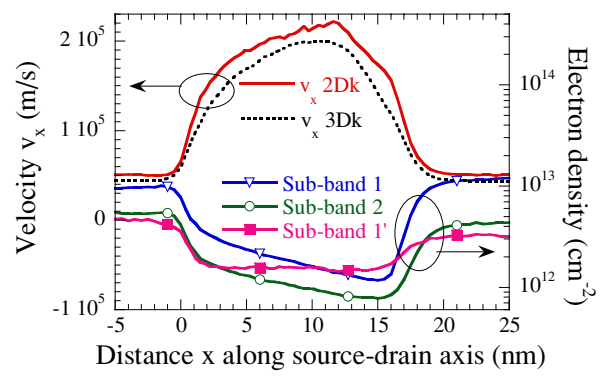

Figure 4. Average velocity $v_{x}$ for both $2 \mathrm{Dk}$ and $3 \mathrm{Dk}$ simulations, and surface density of the first sub-bands versus distance $x$ along the S-D direction at the on-state. N.B: $E_{1}^{\prime}>E_{2}>E_{1}$.

and $\mathrm{Si}$ body $T_{\mathrm{Si}}$ thicknesses are equal to $1.2 \mathrm{~nm}$ and $5 \mathrm{~nm}$, respectively. The doping density is $N_{\mathrm{D}}=5 \times 10^{19} \mathrm{~cm}^{-3}$ in the $N^{+}$source-drain regions and $N_{\mathrm{A}}=2 \times 10^{15} \mathrm{~cm}^{-3}$ in the body. The work function of the gate material is $4.46 \mathrm{eV}$. The supply voltage $V_{\mathrm{DD}}$ is $0.7 \mathrm{~V}$.

Figure 3 indicates that the electron density is strongly modified by the quantization along the $z$-axis. Electrons are moved away from the $\mathrm{Si} / \mathrm{SiO}_{2}$ interface due to quantum repulsion in the whole structure even in the source and drain regions.

Figure 4 shows that the velocities in the channel for both $2 \mathrm{Dk}$ and $3 \mathrm{Dk}$ simulations are much higher than the stationary saturation velocity (about $10^{5} \mathrm{~m} \mathrm{~s}^{-1}$ ). The transport

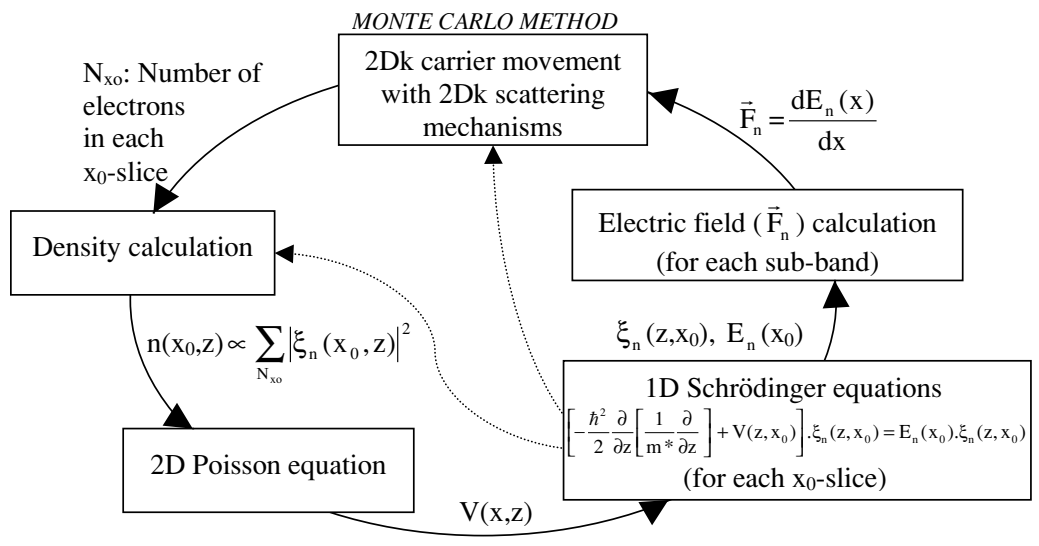

Figure 2. Multi sub-band Monte Carlo algorithm during one time step. 


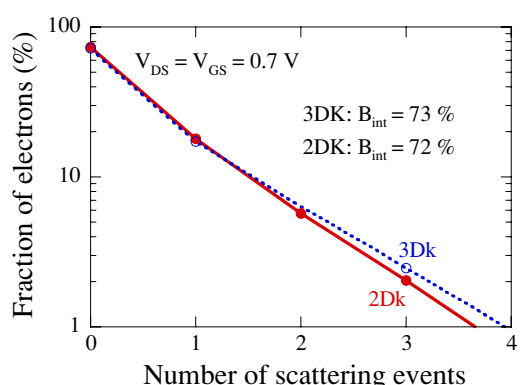

Figure 5. Fraction of electrons versus the number of scattering events undergone at the on-state.

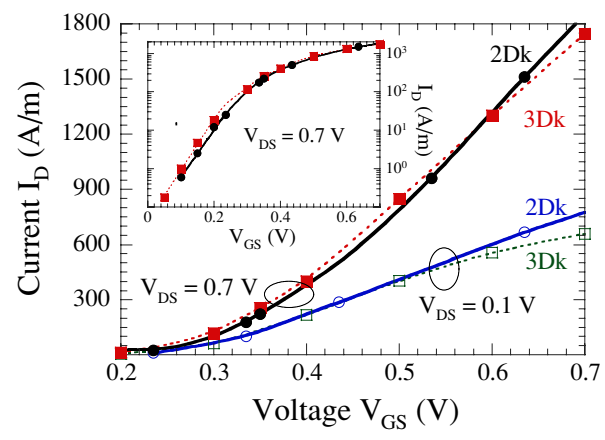

Figure 6. Drain current $I_{\mathrm{D}}$ versus gate voltage $V_{\mathrm{GS}}$ at $V_{\mathrm{DS}}=0.1 \mathrm{~V}$ and $0.7 \mathrm{~V}$.

is therefore far from equilibrium in this $15 \mathrm{~nm}$ long channel. Moreover, figure 4 exhibits hot electron transfer from the lowest energy sub-band to higher sub-bands, in particular near the drain region. Indeed, the first primed sub-band (primed and unprimed sub-bands have a quantization mass of $0.916 \mathrm{~m}_{0}$ and $0.19 m_{0}$, respectively) with high energy $\left(E_{1}^{\prime}>E_{2}>E_{1}\right)$ is less occupied than lower sub-bands in the near-equilibrium source region. However, due to inter sub-band phonon scattering, it becomes relatively more and more populated as the drain is reached.

For both 3Dk and 2Dk simulations at the on-state, we have calculated the number of scattering events $N_{\text {scatt }}$ experienced by each carrier crossing the channel from source-end to drainend. The resulting scattering spectra are plotted in figure 5

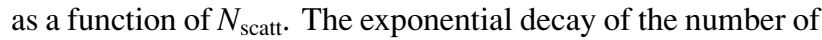
electrons with increasing $N_{\text {scatt }}$ illustrates the quasi-ballistic behaviour of carrier transport. The fraction of ballistic electrons $B_{\text {int }}$ reaches $72 \%$ and $73 \%$ in $2 \mathrm{Dk}$ and $3 \mathrm{Dk}$ curves, respectively. The effect of bulk phonon scattering in the channel is thus similar in 2Dk and 3Dk electron gases.

At last, figure 6 presents the evolution of the drain current as a function of the gate voltage for both $3 \mathrm{Dk}$ and $2 \mathrm{Dk}$ simulations. First, we observe that the current is only softly modified by quantization effects. The subthreshold curves plotted in the inset of figure 6 show a weak threshold voltage shift $\left(\Delta V_{\mathrm{T}}=10 \mathrm{mV}\right)$ and a slight degradation of subthreshold behaviour induced by quantization: the subthreshold slope increases from 75 to $82 \mathrm{mV} / \mathrm{dec}$ for, respectively, 3Dk and 2Dk simulations.

\section{Conclusion}

We have developed a multi sub-band MC simulator for n-MOS transistors that extends the quantum confinement calculation to the whole device region. It also uses a simple and effective selfconsistent iterative method that gives meaningful results with good convergence. The simulation of a $15 \mathrm{~nm}$ long DGMOS reveals that the transport is strongly far from equilibrium which justifies the use of the Monte Carlo approach. Multi sub-band description opens up the possibility of detailed investigation of nanoscale device physics. However, for the $5 \mathrm{~nm}$ thick DGMOS studied in this work, all other things being equal, the $I-V$ characteristics are weakly modifed by quantum confinement.

\section{Acknowledgments}

This work is supported by the French RMNT under project CMOS-D-ALI and by the European Community 6th FP under contract IST 506844 (NoE SINANO).

\section{References}

[1] Saint-Martin J et al 2006 Comparison of multiple-gate MOSFET architectures using Monte Carlo simulation Solid-State Electron. 50 94-101

[2] Hareland S A et al 1996 A computationally efficient model for inversion layer quantization effects in deep submicron N-channel MOSFET's IEEE Trans. Electron Devices 43 90-6

[3] Gamiz F et al 2004 Double gate silicon on insulator transistors. A Monte Carlo study Solid-State Electron. 48 937-45

[4] Esseni D et al 2003 Physically based modeling of low field electron mobility in ultrathin single- and double-gate SOI n-MOSFETs IEEE Trans. Electron Devices 50 2445-55

[5] Saint-Martin J et al 2004 Influence of ballistic effects in ultra-small MOSFETs J. Comput. Electron. 3 207-10

[6] Venugopal R et al 2002 Simulating quantum transport in nanoscale MOSFETs: real vs. mode space approaches J. Appl. Phys. 92 3730-9

[7] Fischetti M V and Laux S E 1993 Monte Carlo study of electron transport in silicon inversion layers Phys. Rev. B 48 2244-74

[8] Dollfus P $1997 \mathrm{Si} / \mathrm{Si}_{1-x} \mathrm{Ge}_{x}$ heterostructures: electron transport and field-effect transistor operation using Monte Carlo simulation J. Appl. Phys. 82 3911-6

[9] Monsef F et al 2004 Electron transport in $\mathrm{Si} / \mathrm{SiGe}$ modulation-doped heterostructures using Monte Carlo simulation J. Appl. Phys. 953587 